\title{
Zika virus vaccines: potentiality and prospects
}

Volume 5 Issue 4 - 2017

\section{Opinion}

Zika virus (ZIKV) remains an public health challenge with requisition of intensive action although the recent declaration of Public Health Emergency of International Concern (PHEIC) by the World Health Organization (WHO) Director-General on November 18, 2016. With possible continuation of the outbreaks, the WHO/ UNICEF have established Zika Virus Vaccine Target Product Profile (TPP) as a strategy to fight against Zika virus infection and disease, particularly prenatal Zika virus infection accompanying with the WHO Zika Strategic Response plan. WHO SAGE would advise at least two scenarios of the immunization strategies: 1. Outbreak response and 2. Routine or endemic transmission use. The City College of New York and a drug company in New York City conducted a Zika virus vaccine against the mosquito-borne Zika virus focusing on virus-like particle (VLP) in animal models and demonstrated the preclinical results with favorable outcomes. The studied VLP vaccine formulations tested in animals can elicit the protective antibodies with neutralizing activity higher than or equivalent to the activity present in recovered Zika-virus-infection patient's serum with good tolerance and good safety. The National Institute of Allergy and Infectious Diseases (NIAID) is developing a vaccine, called "AGS-v "which designed to protect against multiple mosquito-borne diseases, including Zika virus infection. AGS-v candidate with being evaluated in a Phase I clinical trial at the National Institute of Health (NIH) Clinical Center in Bethesda, Maryland, triggers an immune response to mosquito salivary proteins from mosquito salivary gland. NIAID is also developing several other Zika virus vaccine candidates, such as a live-attenuated investigational vaccine designed to protect against Zika virus infection and dengue virus infection, which is closely related to a dengue vaccine candidate presently being evaluated in a large Phase III study in Brazil. A monovalent vaccine candidate who is designed to protect only against Zika virus will enter a Phase I trial at University of Vermont and Johns Hopkins University in Baltimore. An investigational Zika vaccine which uses a genetically engineered version of vesicular stomatitis virus, an animal virus that primarily affects cattle (VSV) was successfully used in an investigational Ebola vaccine developed by NIAID. This VSV vaccine candidate is underway to evaluate the vaccine candidate in tissue culture and animal models. NIAID is co-funding the Phase I clinical trials program with the Walter Reed Army Institute of Research (WRAIR), serving as the regulatory sponsor for several of these clinical trials and providing other support in developing a purified inactivated Zika vaccine, called "ZPIV". Four of five Phase I trials for ZPIV testing have launched at the WRAIR Clinical Trial Center in Silver Spring, Maryland, clinical research center "CAIMED” (part of Ponce Health Sciences University in Puerto Rico), the Center for Virology and Vaccine Research (part of Beth Israel Deaconess Medical Center and Harvard Medical School in Boston), and the Center for Vaccine Development at the Saint Louis University School of Medicine. A Deoxyribonucleic Acid (DNA)based Zika vaccine candidate developed at NIAID's Vaccine Research Center (VRC) is similar to the VRC's investigational flavivirus vaccine for West Nile virus infection. This vaccine candidate entered a Phase I clinical trial at NIAID in August 2016. A second Phase I trial and a

\author{
Attapon Cheepsattayakorn, ${ }^{1,2,3}$ Ruangrong \\ Cheepsattayakorn ${ }^{4}$ \\ 'Editor-in-Chief, Journal of Human Virology and Retrovirology, \\ USA \\ ${ }^{2}$ Oth Zonal Tuberculosis and Chest Disease center, Thailand \\ ${ }^{3}$ Department of Disease Control, Ministry of Public Health, \\ Thailand \\ ${ }^{4}$ Department of Pathology, Faculty of Medicine, Chiang Mai \\ University, Thailand
}

Correspondence: Attapon Cheepsattayakorn, I0th Zonal Tuberculosis and Chest Disease Center, I 43 Sridornchai Road Changklan Muang, Chiang Mai 50I00, Thailand, Tel 6653 I40767, 6653 276364, Fax 6653 140773, 6653273590 .

Email attapon1958@gmail.com, attaponche@yahoo.com

Received: May 27, 2017 | Published: May 29, 2017

Phase II clinical trial were launched in December 2016 and March 2017, respectively. Sites of a Phase II clinical trial included Houston, Peru, Puerto Rico, Costa Rica, Brazil, Mexico and Panama. This twopart trial, called "VRC 705", will further evaluate the immunogenicity and safety of this vaccine candidate. NIAID ' $\mathrm{s}$ VRC is collaborating with the University of Pennsylvania and the two drug companies to evaluate several investigational messenger-Ribonucleic Acid (mRNA) vaccine candidates (a gene-based platform similar to DNA vaccine candidates). An investigational mRNA vaccine candidate is being evaluated in a Phase I trial, whereas another vaccine candidate could be entered clinical trials in late 2017 .

A previous study on a live-attenuated Zika virus vaccine candidate in mouse model revealed that a live-attenuated Zika virus vaccine candidate containing a 10 -nucleoside deletion in the $3^{\prime}$ translated region of the Zika virus genome (10-del ZIKV) can be highly attenuated, immunogenic, and protective in type 1 interferon receptor-deficient A129 mice. This attenuated 10-del ZIKV was incapable of infecting mosquitoes after oral feeding of spiked blood meals, representing an additional safety property. Two recent studies reported the successful experimental ZIKV vaccine candidates testing in animal models, one by Richner et al. and another by Pardi et al. Both vaccine candidates were engineered at the mRNAs with sequences encoding the ZIKV precursor membrane (prM) glycoprotein and envelop (E) glycoprotein, which is critical to viral attachment, entry, and replication in the infected host cells. In conclusion, it is currently still unclear about the role of potentially cross-reactive antibodies in disease enhancement or prevention. Although several in vitro studies have revealed both potentially cross-reactive antibodies in disease enhancement or prevention, the generalization of these studies to the clinical relevance and human immunological experience is impossible. Preliminary data in flavivirus primed non-human primates do not demonstrate any features of enhanced disease. However, it is critical area for further field evaluation. 
Acknowledgments

None.

\section{Conflicts of interest}

None. 